\title{
ON SYSTEMS OF SELF-CONSISTENT ABSTRACTIONS
}

\author{
YOSHINOBU INOUE
}

In this paper, I would like to show that there are many systems of abstractions, each abstraction being self-consistent, but each system as a whole leading to contradiction or to some pathological situation.

Prof. K. Ono gave me a preprint of his recent work "MUTUAL CONTRADICTION OF TWO SELF-CONSISTENT ABSTRACTIONS" and suggested me to study further along this direction.

Any relation $Q(x, \ldots, z)$ is called TF-invariant if and only if it takes the same truth value for the following two truth-value evaluations: all the elementary formulas in $Q(x, \ldots, z)$ are evaluated true (false).

Now, let $\subseteq$ be any set of relations then any abstraction of the form
(A)
$(E p)(x)(x \in p \equiv \mathfrak{A}(x))$

is called an $\mathfrak{\Xi}$-abstraction if and only if $\mathfrak{U}(x)$ is expressible exclusively in terms of relations in $\subseteq$. If $₫$ is a set of TF-invariant relations, then any $\Im$-abstrac tion is proved self-consistent. These abstractions, however, may contradict to each other ${ }^{1)}$.

In (1), I have generalized Ono's result as follows : Let

$$
(E p)(x)(x \in p \equiv R(x))
$$

be any self-contradictory abstraction, then,

$$
Q(x, y, z) \equiv \cdot x \in y \wedge R(z)
$$

is a TF-invariant relation. Accordingly if we define $x \in \underset{R}{\in} y$ by $Q(x, y, x)$, any $\left\{\frac{\epsilon}{\bar{R}}\right\}$-abstraction must be self-consistent but the whole class of such abstractions leads to contradiction.

Ono and Ohta gave a sufficient condition on $R$ of (B) for that (B) is self-

Received January 28, 1966.

1) See Ono [2]. 
contradictory ${ }^{2}$.

In (2), I will show the following: If we define $x \stackrel{R}{\in} y$ by $Q(x, y, y)$ for any $R$ satisfying the condition, not only any $\{\stackrel{R}{=}\}$-abstraction is self-consistent, but also the whole class of $\{\stackrel{R}{\in}\}$-abstractions can be proved consistent. However, the class of $\{\stackrel{R}{\in}\}$-abstractions leads to pathological situation that all the sets become empty in a certain sense.

At the 1965 Annual Meeting of The Mathematical Society of Japan, Prof. Ono spoke on a class of restricted abstractions, each being self-consistent, and he proposed to examine the system further because he could develop the essential part of set theory starting from the restricted abstractions only ${ }^{3)}$. On investigating his system carefully, I have realized that his system is contradictory.

In (3), I will give a proof of contradiction after giving a sketch of his system. The proof is given rather formally adopting the practical way of description introduced by Ono ${ }^{4}$. I wish to express my thanks to Prof. Ono for his kind guidance.

\section{Mutual contradiction of $\{\underset{R}{\epsilon}\}$-abstractions}

Let $R(x)$ be a condition on $x$ for which the abstraction

$$
(E p)(x)(x \in p \equiv R(x))
$$

causes a Russell-type paradox. Let $\in_{\boldsymbol{R}}$ be a relation defined by

$$
(1.1 \mathrm{D})^{5)} \quad x \in \in_{R} y \equiv \cdot x \in y \wedge R(x) .
$$

The relation $\underset{B}{\in}$ is a special case of $Q$ of (C) (i.e. $x \underset{R}{\in} y \equiv Q(x, y, x)$.), therefore any $\left\{\in_{R}\right\}$-abstraction is self-consistent.

$$
\left\{\in_{B}\right\} \text {-abstractions contradict to each other. }
$$

Proof. Because the $\{\underset{R}{\in}\}$-abstraction

2) Ono and Ohta spoke on the matter at the 1965 Annual Meeting of the Mathematical Society of Japan, but they have not yet published their result. Exact description of the sufficient condition will be given later.

3) Prof. Ono gave me his manuscript of his development of set theory, which would not be published.

4) See Ono [1].

5) Symbols $\mathrm{D}, \mathrm{A}, \mathrm{T}$, and $\mathrm{R}$ of numberings of the forms $(m . n \mathrm{D}),(m . n \mathrm{~A}),(m . n \mathrm{~T})$, and $(m . n \mathrm{R})$ denote that $(m . n \mathrm{D}),(m . n \mathrm{~A}),(m . n \mathrm{~T})$, and $(m . n \mathrm{R})$ are a DEFINITION, an AXIOM SCHEME, a THEOREM and a REMARK, respectively. 


$$
(E s)(x)(x \in s \equiv \cdot x \underset{\bar{R}}{\in} x \rightarrow x \underset{\bar{R}}{\leftarrow} x)
$$

guarantees existence of an object $s$ for which $(x) x \in s$ holds, $(E y) x \underset{R}{\leftarrow} y$ is equivalent to $R(x)$. Consequently, the $\{\underset{\bar{R}}{\in}\}$-abstraction

$$
(E p)(x)(x \in p \equiv(E y) x \underset{\bar{R}}{\in} y)
$$

is equivalent to the paradoxical abstraction

$$
(E p)(x)(x \in p \equiv R(x))
$$

\section{On $\{\stackrel{R}{E}\}$-abstractions}

Let $R(y)$ be a predicate which satisfies following conditions (F) and (G) simultaneously:

$$
\begin{aligned}
& y \in y \rightarrow \rightarrow R(y) \\
& -R(y) \rightarrow(E z)(z \in y \wedge \rightarrow R(z))^{6}
\end{aligned}
$$

then, (B) is self-contradictory for this $R$. Now let $\stackrel{R}{E}$ be the ralation defined by

$$
x \stackrel{R}{\in} y \rightleftharpoons \cdot x \in y \wedge R(y)
$$

Since relation $\stackrel{R}{\epsilon}$ can be regarded as a special case of $Q$ of (C) (i.e. $x \stackrel{R}{\in} y \equiv Q(x, y, y)$ assuming that $R(y)$ satisfies $(\mathrm{F})$ and $(\mathrm{G})$.), any $\{\stackrel{R}{E}\}$-abstraction is self-consistent.

$$
\{\stackrel{R}{\in}\} \text {-abstractions are mutually consistent. }
$$

Proof. Let us define a binary relation $\in$ for two objects 0 and 1 by following stipulations :

$$
0 \notin 0, \quad 1 \notin 0, \quad 0 \in 1, \quad 1 \in 1 .
$$

Since $-R(1)$ holds by (F) and 0 has no member, $u \stackrel{R}{=} v$ is always false by $(2.1 \mathrm{D})$. Consequently, any sentence $\mathfrak{H}(x)$ which is expressible in terms of $\stackrel{R}{E}$ only is always false or always true. So the $\{\stackrel{R}{\in}\}$-abstractions (A) hold, if we take 0 and 1 in place of $p$ of $(A)$ in the respective case.

\section{$(2.3 \mathrm{~T})(\mathrm{Er})(E s) s \stackrel{R}{E} r$ and $\{\stackrel{R}{E}\}$-abstractions contradict mutually.}

6) Sufficient condition of Ono-Ohta for (B) to be self-contradictory. See foot-note ${ }^{2)}$. 
Proof. $/ \mathrm{A} \rightarrow \mathrm{d}$, i.
A) $(E r)(E s) s \stackrel{R}{\in} r$.
b) $E r !(E s) s \stackrel{R}{E} r / \mathrm{A}$.
c) $E p ! \quad(x)(x \in p \equiv(E y) y \stackrel{R}{E} x) \quad /(\mathrm{A})$.
d)) $\quad R(p) \quad / \mathrm{dA} \rightarrow \mathrm{dd}, \mathrm{bd}$.
$\mathrm{dA}) \quad \rightarrow R(p)$.
$\mathrm{db}) E t ! \quad t \subseteq p \wedge \rightarrow R(t) \quad / \mathrm{dA},(\mathrm{G})$.
dc) $E y ! \quad y \stackrel{R}{\in} t / \mathrm{db}, \mathrm{c}$.
dd) $R(t) / \mathrm{dc},(2.1 \mathrm{D})$.
e) $r \in p / b, c$.
f) $r \stackrel{R}{=} p / \mathrm{d}$, e.
g) (Ey)y $\stackrel{R}{\in} p$ /f.
h) $p \in p / g, c$.
i) $\quad \rightarrow R(p) \quad / \mathrm{h},(\mathrm{F})$.

Let us now define the relation $=$ as usual by

$$
x=y \rightleftharpoons(s)(s \in x \equiv s \in y) .
$$

Then binary relation $=$ is surely $\mathrm{TF}$-invariant. So any $\{\stackrel{R}{E},=\}$-abstraction is self-consistent.

However, we can deduce easily $(E r)(E s) \stackrel{R}{\stackrel{R}{=}} r$ from the pair of $\{=\}$-abstractions

$$
(E s)(x)(x \in s \equiv \cdot x=x \wedge x \neq x)
$$

and

$$
(E r)(x)(x \in r \equiv x=s) .
$$

According to $(2.3 \mathrm{~T})$, this result shows that some $\{\stackrel{R}{\epsilon},=\}$-abstractions contradict mutually.

$(2.5 \mathrm{R})(2.2 \mathrm{~T})$ holds even if we replace the conditions $(\mathrm{F})$ and $(\mathrm{G})$ by the following condition :

Not only $(E p)(y)(y \in p \equiv R(y))$ but also at least one of

$$
\begin{aligned}
& (E p)(y)(y \in p \equiv \cdot R(y) \vee y \notin y) \text { and }(E p)(y)(y \in p \equiv \cdot R(y) \wedge y \notin y) \\
& \text { are self-contradictory. }
\end{aligned}
$$

This can be confirmed by the model employed in (2.2 T). $R(y)$ surelv satisfies condition $(H)$ if it does $(F)$ and $(G)$.

(2.6 R) We can not assert $(2.3 \mathrm{~T})$ if $R(y)$ is the kernel of a self-contradictory abstraction but does not satisfy $(F)$ and $(G)$ simultaneously. In fact, $\{\stackrel{R}{=}\}$-abstractions and $(E u)(E v) u \stackrel{R}{=} v$ are mutually consistent when we take $R(y)$ as $(E z)(z \notin z \notin y)$. Namely, both $\{\stackrel{R}{=}\}$-abstractions and $(E u)(E v) u \stackrel{R}{=} v$ are satisfied 
simultaneously in the class $\{0, p, q, 1\}$ where the relation $\in$ is defined as follows :

$0, p, q, 1 \notin 0, \quad p \in p, \quad 0, q, 1 \notin p, \quad 0, q, 1 \in q, \quad p \notin q$ and $0, p, q, 1 \in 1$.

\section{Contradiction of Ono's system ${ }^{7)}$}

The system proposed by Ono is a formal system standing on the lower classical predicate logic having membership $\in$ as its sole primitive notion. This system assumes only one axiom scheme, $\{\subset, \underset{0}{\in}\}$-abstractions. In the following, I will prove that this system is contradictory after introducing the system formally.

(3.1 D) INCLUSION $x \subset y \equiv(s)(s \in x \rightarrow s \in y)$.

(3.2 D) STRICT-MEMBERSHIP $x \in y \rightleftharpoons \cdot x \in y \wedge y \nsubseteq x$.

(3.3 A) AXIOM SCHEME The axiom scheme of this system is $\{\in \in, \subset\}$ abstractions, i.e.

$$
(E p)(x)(x \in p \equiv \mathfrak{A}(x)) .
$$

where $\mathfrak{A}(x)$ is expressible in terms of inclusion $\subset$ and strict-membership $€$ only.

(3.4 D) Let us call any sentence, any predicate, or any relation PROPER if and only if they are expressible in terms of inclusion $\subset$ and strict-membership $\lesssim$ only.

(3.5 R) Inclusion $\subset$ and strict-membership $\underset{0}{\in}$ are TF-invariant. Hence, every axiom of this system is self-consistent.

(3.6 D) EQUALITY $x=y \equiv(s)(s \in y \equiv s \in x)$.

(3.7 T) Inclusion $\subset$ is reflexive and transitive. Equality $=$ is reflexive, symmetric and transitive.

$(3.8 \mathrm{~T}) \quad x=y \equiv \cdot x \subset y \wedge y \subset x, \quad x \in \subset y \equiv x \in y$.

(3.9 T) $x \in y \rightarrow x \in y, \quad x \in y \rightarrow y \mp x$.

(3.10 D) NULL OBJECT $\varnothing(p) \equiv \rightarrow(E s) s \in p$.

$(3.11 \mathrm{~T})(E x) \varnothing(x) \quad($ By $(3.3 \mathrm{~A})$.$) .$

7) See foot-note ${ }^{3)}$. 
$(3.12 \mathrm{~T}) \quad \emptyset(p) \equiv(x) p \subset x \quad(\mathrm{By}(3.1 \mathrm{D}),(3.8 \mathrm{~T}),(3.10 \mathrm{D})$ and $(3.11 \mathrm{~T})$.$) .$

$(3.13 \mathrm{R})$ According to $(3.12 \mathrm{~T}) \varnothing$ can be regarded as proper.

(3.14 D) STRICT NULL OBJECT $\varnothing^{*}(p) \equiv-(E s) s \in$

(3.15 D) REGULAR OBJECT

$$
\mathfrak{N}(x) \risingdotseq\left(E y !\left(x=\epsilon_{0} y \wedge(z)\left(z \subset y \wedge \emptyset^{*}(z) \cdot \rightarrow \emptyset(z)\right)\right) .\right.
$$

$(3.16 \mathrm{R})$ The predicate $\Re(x)$ is proper by $(3.1 \mathrm{D}),(3.4 \mathrm{D}),(3.6 \mathrm{D}),(3.13 \mathrm{R})$ and $(3.14 \mathrm{D})$.

(3.17 R) The predicate $\Re(x)$ is almost equivalent to the predicate of $x \notin x$. Actually, we can easily prove by making use of $(E y)(s)(s \in y \equiv s=x)$ that $\neg x=\epsilon x$ implies $\mathfrak{R}(x)$.

$(3.18 \mathrm{~T}) \quad x=y \wedge \mathfrak{R}(x) \cdot \rightarrow \mathfrak{R}(y) \quad(\mathrm{By}(3.15 \mathrm{D}))$.

$(3.19 \mathrm{~T}) \quad(s)(s \in p \rightarrow \Re(s)) \cdot \rightarrow \mathfrak{R}(p)$.

Proof. $/ \mathrm{A} \rightarrow \mathrm{b}$.

A) $(s)(s \in p \rightarrow \mathfrak{R}(s))$.

b)） $\Re(p) \quad$ bA $\rightarrow$ bi. bA) $\rightarrow \mathfrak{R}(p)$.

bb) Ey! $(t)(t \in y \equiv t=p) \quad /(3.3 \mathrm{~A}),(3.8 \mathrm{~T})$.

bc) $p \in y \quad / \mathrm{bb},(3.7 \mathrm{~T})$.

bd)） $y \leftarrow p \quad(\mathrm{bdA} \rightarrow \mathrm{bdc}, \mathrm{bA} . \quad$ bdA $\quad y \subset p$.

bdb) $\quad p \in p \quad / \mathrm{bdA}, \mathrm{bc},(3.8 \mathrm{~T}) . \quad$ bdc $) ~ \Re(p) \quad / \mathrm{bdb}, \mathrm{A}$.

be) $p \overline{\bar{o}} y / \mathrm{bc}, \mathrm{bd}$. bf) $p=\epsilon_{\overline{0}} y \quad / \mathrm{be},(3.7 \mathrm{~T})$.

$\mathrm{bg})) \quad(z)\left(z \subset y \wedge \emptyset^{*}(z) . \rightarrow \varnothing(z)\right) \quad / \mathrm{bgA} \rightarrow$ bgb.

bgA) $A z ! \quad z \subset y \wedge \varnothing^{*}(z)$.

bgb)) $\quad((z) \quad$ bgbA $\rightarrow$ bgbg, bgA. bgbA) $\rightarrow \varnothing(z)$.

bgbb) Es! $s \in z \quad$ /bgbA. bgbc) $s \in y$ /bgA, bgbb.

bgbd) $s=p \quad / \mathrm{bgbc}, \mathrm{bb}$.

bgbe)) $z \mp s \quad$ /bgbeA $\rightarrow$ bgbee, bA. bgbeA) $z \subset s$.

bgbeb) $s \in s$ /bgbeA, bgbb, (3.8 T).

bgbec) $s \in p$ /bgbeb, bgbd, (3.8 T).

bgbed) $\Re(s)$ /A, bgbec. bgbee) $\Re(p)$ /bgbed, bgbd, (3.18 T).

bgbf) $s \equiv z / \mathrm{bgbb}$, bgbe, (3.2 D).

bgbg) $\rightarrow 0 *(z)$ /bgbf, (3.14 D). 
bh) $\quad(E y)\left(p=\epsilon_{0} y \wedge(z)\left(z \subset y \wedge \emptyset^{*}(z) \cdot \rightarrow \emptyset(z)\right)\right) \quad /$ bg, bf, bb.

bi) $\mathfrak{R}(p) / \mathrm{bh},(3.15 \mathrm{D})$.

$(3.20 \mathrm{~T})$

$$
\neg(E p)(x)(x \in p \equiv \Re(x)) .
$$

Proof. / A $\rightarrow \mathrm{h}, \mathrm{j}$.
A) $(E p)(x)(x \in p \equiv \Re(x))$.
b) $E p$ ! $(x)(x \in p \equiv \Re(x)) \quad / \mathrm{A}$.
c) $\mathfrak{x}(p) / \mathrm{b},(3.19 \mathrm{~T})$.
d) Er! Ey! $p=r \in_{0} y \wedge(z)\left(z \subset y \wedge \emptyset^{*}(z) . \rightarrow \emptyset(z)\right) \quad / c,(3.15 \mathrm{D})$.
e) $E z ! \quad(s)\left(s \in z \equiv \cdot s=r \wedge(t)\left(s \equiv_{0} t \equiv r \in t\right)\right) \quad /(3.3 \mathrm{~A})$.
f) ) $z \subset y \quad / \mathrm{fA} \rightarrow \mathrm{fd},(3.1 \mathrm{D}) . \quad$ fA) $\quad A s ! \quad s \in z$.
fb) $(t)\left(s \in t \equiv r \in_{0} t\right) \quad$ /fA, e. fc) $s \in y \quad / \mathrm{fb}, \mathrm{d}$.
fd) $s \in y \quad / \mathrm{fc},(3.9 \mathrm{~T})$.
g)) $\quad \emptyset^{*}(z) \quad$ gA $\rightarrow$ ge, gf. gA) $\quad \rightarrow \emptyset^{*}(z)$.
gb) $E s ! \quad s \in z \quad / \mathrm{gA},(3.14 \mathrm{D}) . \quad \mathrm{gc}) \quad(t)(s \in t \equiv r \in t) \quad / \mathrm{gb}, \mathrm{e},(3.9 \mathrm{~T})$.
gd) $\quad r \Xi_{0} z / \mathrm{gb}, \mathrm{gc}$. ge) $z \leftarrow r / \mathrm{gd},(3.2 \mathrm{D})$.
gf)) $z \subset r \quad$ (gfA $\rightarrow$ gff. $\quad$ gfA) $A s ! \quad s \in z$.
gfb ) $s=r / g f A$, e. $\quad$ gfc $) ~ s=p=r / g f b, d,(3.7 \mathrm{~T})$.
gfd) $\quad i(s) \quad / g f c, c,(3.18 \mathrm{~T}) . \quad$ gfe $) \quad s \in p \quad / \mathrm{b}$, gfd.
gff) $s \in r$ /gfe, gfc, $(3.8 \mathrm{~T})$.
h) $\quad \varnothing(z) / d, f, g . \quad$ i) $\quad r \in z \quad / \mathrm{e},(3.7 \mathrm{~T})$.
j) $\rightarrow \varnothing(z) / \mathrm{i},(3.10 \mathrm{D})$.
$(3.21 \mathrm{~T})$
$(E p)(x)(x \in p \equiv \Re(x))$.

This is one of $\left\{\epsilon_{0}, \subset\right\}$-abstractions, for $\Re(x)$ is proper according to (3.16 R). $(3.21 \mathrm{~T})$ and $(3.20 \mathrm{~T})$ contradict to each other.

\section{REFERENCES}

[1] Ono, K., On a practical way of describing formal deductions, Nagoya Math. Jour., vol. 21 (1962), pp. 115-121.

[2] Ono, K., Mutual contradiction of two self-consistent abstractions, Nagoya Math. Jour., vol. 28 (1966), pp. 59-62.

[3] Ono, K., A certain kind of formal theories, Nagoya Math. Jour., vol. 25 (1965), pp. $59-86$.

\section{Mathematical Institute,}

Nagoya University 